\section{Associações entre consumo de produtos lácteos, proteína C-reativa e perfil lipídico em adultos: resultados do ELSA-Brasil}

\author{
Associations between consumption of dairy \\ products, C-reactive protein, and lipid profile \\ in adults: results of the ELSA-Brasil study
}

\author{
Asociaciones entre consumo de productos \\ lácteos, proteína C-reativa y perfil lipídico \\ en adultos: resultados del ELSA-Brasil
}

\author{
Amanda Gomes Ribeiro 1 \\ José Geraldo Mill 1 \\ Sheila Maria Alvim Matos 2 \\ Gustavo Velasquez-Melendez 3 \\ Nágela Valadão Cade 1 \\ Maria del Carmen Bisi Molina 1
}

doi: 10.1590/0102-311X00028019

\title{
Resumo
}

Anormalidades lipídicas e inflamação sistêmica subclínica estão associadas ao processo de aterosclerose, sendo utilizadas como marcadores de risco cardiovascular. Estudos sugerem um possível efeito benéfico dos produtos lácteos na saúde cardiovascular, mas os resultados em marcadores lipídicos e inflamatórios ainda são controversos. O objetivo deste trabalho foi avaliar a associação entre o consumo de produtos lácteos e seus diferentes subgrupos e proteina $C$-reativa $(P C R), L D L$-colesterol $(L D L-C)$ e razão triglicerídeo/HDL-colesterol (TG/HDL-C) nos participantes do Estudo Longitudinal de Saúde do Adulto (ELSA-Brasil) $(n=9.372)$. O consumo de lácteos foi avaliado por meio de questionário de frequência alimentar validado e apresentado em porções/dia. O consumo total de lácteos foi descrito em quatro categorias $(\leq 1$ porção/dia a > 4 porções/dia). As associações foram estimadas por meio do odds ratios $(O R)$, utilizando-se o grupo de menor consumo ( $\leq 1$ porção/dia) como referência. Os menores valores de OR para TG/HDL-C no modelo multivariado (0,70; IC95\%: 0,55-0,90 em homens; e 0,55; IC95\%: 0,43-0,70 em mulheres) foram encontrados no grupo com consumo $>4$ porções/dia de lácteos totais. Esses resultados foram apoiados pelas associações inversas encontradas entre diferentes subgrupos de lácteos e a razão TG/HDL-C. Não foi encontrada associação entre consumo de produtos lácteos e seus subgrupos e valores de LDL-C e de PCR. Os resultados sugerem um possível efeito benéfico dos lácteos no perfil lipídico, porém são necessárias evidências de estudos longitudinais e de intervenção que elucidem os mecanismos de efeito dos diferentes tipos de lácteos.

Laticinios; Inflamação; Doenças Cardiovasculares; Dislipidemias

\author{
Correspondência \\ M. C. B. Molina \\ Universidade Federal do Espírito Santo. \\ Av. Marechal Campos 1468, Vitória, ES 29047-105, Brasil. \\ mdcarmen2007@gmail.com \\ 1 Universidade Federal do Espírito Santo, Vitória, Brasil. \\ 2 Universidade Federal da Bahia, Salvador, Brasil. \\ 3 Universidade Federal de Minas Gerais, Belo Horizonte, Brasil.
}




\section{Introdução}

Segundo dados da Organização Mundial da Saúde (OMS), as doenças cardiovasculares (DCV) são responsáveis por um terço da mortalidade em todo o mundo. A cardiopatia isquêmica, o acidente vascular cerebral (AVC), a hipertensão arterial e outras DCV causam cerca de 17 milhões de óbitos anualmente, representando um grande desafio à saúde pública 1 . As doenças isquêmicas do coração e as doenças cerebrovasculares constituem a manifestação predominante, representando dois terços dos óbitos por DCV. Ambas têm como principal causa básica a aterosclerose 2.

As lipoproteínas de baixa densidade (LDL-C) desempenham um papel central no desenvolvimento da aterosclerose 3 , sobretudo partículas de LDL-C pequenas e densas (fenótipo B), com maior potencial aterogênico. Evidências indicam que valores elevados de triglicerídeos (TG) plasmáticos estariam associados ao aumento das LDL-C pequenas e densas, bem como à redução das lipoproteínas de alta densidade (HDL-C) ${ }^{4}$, o que caracterizaria um conjunto de anormalidades lipídicas chamado de "fenótipo de lipoproteína aterogênica" ou "dislipidemia aterogênica" 5 . Dessa forma, estudos têm associado maiores valores da razão TG/HDL-C à predominância do fenótipo B de LDL-C 6,7 e sugerido seu uso como um marcador independente de risco cardiovascular 8,9 .

O efeito dos lácteos sobre os lipídeos plasmáticos ainda é controverso, tendo em vista os resultados provenientes de diferentes desenhos de trabalhos 10. Um estudo observacional com 130.420 adultos coreanos mostrou uma relação inversa entre maior frequência de consumo de leite e hipertrigliceridemia, bem como com baixos valores de HDL-C 11. Huo Yung Kai et al. 12, analisando dados de uma amostra de 3.078 adultos franceses, encontraram uma associação inversa entre o consumo elevado de produtos lácteos com o baixo teor de gordura e LDL-C, mas nenhuma relação com HDL-C ou TG, e nenhum dos parâmetros lipídicos foi associado ao consumo de lácteos integrais. Por outro lado, um ensaio clínico randomizado conduzido por Nilsen et al. 13 mostrou, após oito semanas de intervenção, uma relação inversa entre o consumo de queijo com alto teor de gordura e valores de TG e colesterol total (CT) em participantes com síndrome metabólica.

Além do perfil lipídico, a proteína C-reativa (PCR), importante marcador de inflamação, também tem sido associada à progressão da aterosclerose ${ }^{4}$. Sua avaliação tem sido recomendada em diferentes guidelines como parâmetro complementar à classificação do risco cardiovascular 14 .

Estudos epidemiológicos indicam que um padrão alimentar caracterizado pelo consumo elevado de cereais integrais e lácteos foi inversamente associado à PCR em adultos com síndrome metabólica 15,16,17,18,19. Na coorte ATTICA foi mostrada uma associação inversa entre consumo de laticínios e níveis plasmáticos de PCR, interleucina 6 (IL-6) e fator de necrose tumoral alfa (TNF- $\alpha$ ) 16. O consumo de 3 porções/dia de lácteos com baixo teor de gordura diminuiu os níveis de TNF- $\alpha$ e proteína de quimioatração de monócitos (MCP-1) em mulheres com síndrome metabólica 17. Porém, em diversos ensaios clínicos randomizados não foi possível mostrar um efeito significativo dos diferentes tipos de lácteos em biomarcadores de inflamação 19 . No entanto, Bordoni et al. 18 , em revisão sistemática de estudos clínicos, observaram efeitos benéficos do consumo de lácteos, sobretudo os fermentados, em indivíduos com desordens metabólicas.

Considerando o papel dos marcadores inflamatórios e do perfil lipídico na predição de DCV, o presente trabalho teve como objetivo avaliar a associação entre o consumo de produtos lácteos e seus diferentes subgrupos e níveis circulantes de PCR, LDL-C e razão TG/HDL-C nos participantes do Estudo Longitudinal de Saúde do Adulto (ELSA-Brasil).

\section{Método}

\section{População do estudo}

Este estudo utilizou dados da linha de base do ELSA-Brasil, uma coorte multicêntrica. Entre os anos 2008 e 2010, foram recrutados e avaliados 15.105 adultos (35-74 anos) de ambos os sexos, todos servidores públicos ativos ou aposentados de universidades e instituições de pesquisa de seis capitais brasileiras (Salvador, Belo Horizonte, Rio de Janeiro, São Paulo, Vitória e Porto Alegre). 
Detalhes sobre a metodologia do estudo foram descritos por Aquino et al. 20 e Schmidt et al. 21. Todos os servidores na faixa etária do estudo eram elegíveis, e o recrutamento foi realizado por intermédio de cartazes, correspondência e contato telefônico. Os participantes foram entrevistados por meio de questionários padronizados que forneciam informações sobre características sociodemográficas, história médica, hábitos de saúde e estilo de vida, e submetidos a avaliações clínicas e laboratoriais.

Para o presente trabalho foram excluídos os participantes com DCV autorreferida $(\mathrm{n}=1.001)$, cirurgia bariátrica $(n=100)$, diabéticos $(n=2.590)$ e em uso de medicação hipolipemiante $(n=1.039)$. Indivíduos com consumo calórico estimado $<500$ ou $>6.000 \mathrm{kcal} / \mathrm{dia}(\mathrm{n}=292)$ ou com valores de PCR $\geq 10 \mathrm{mg} / \mathrm{L}(\mathrm{n}=325)$ também foram excluídos. Dos 9.758 participantes restantes, 9.372 possuíam dados completos para as análises.

\section{Dados dietéticos e consumo de lácteos}

Para a obtenção dos dados dietéticos foi utilizado um questionário de frequência alimentar (QFA) semiquantitativo validado para a população do estudo, que incluía 114 itens alimentares para a avaliação do consumo habitual nos últimos 12 meses 22 .

Nove categorias de frequência de consumo foram incluídas, variando desde "Mais de 3x/dia" até "Nunca/Quase nunca”. Para cada alimento/preparação, a média de ingestão diária (g/dia) foi calculada considerando-se a frequência e quantidade reportada de consumo.

Foram considerados os produtos lácteos: leite (integral, desnatado e semidesnatado); iogurte (integral e reduzido teor de gordura); queijo e requeijão (integral e reduzido teor de gordura) e manteiga. Foi calculado o consumo em porções/dia para o total de produtos lácteos e dos subgrupos: produtos lácteos integrais (leite, queijo e iogurte integrais) e com reduzido teor de gordura (leite desnatado e semidesnatado, iogurte e queijo com baixo teor de gordura), lácteos fermentados (iogurte e queijo), leite total, iogurte, queijo e manteiga. Foram consideradas as quantidades por porção para cada item: $240 \mathrm{~g}$ para leite, $120 \mathrm{~g}$ para iogurte, $30 \mathrm{~g}$ para queijo e requeijão e $5 \mathrm{~g}$ para manteiga. Os lácteos totais foram categorizados em quatro grupos de consumo: $\leq 1$ porção/dia; $>1-2$ porções/dia; $>2-4$ porções/ dia e $>4$ porções/dia.

\section{Perfil lipídico e PCR}

Os procedimentos de coleta das amostras biológicas foram padronizados para garantir a uniformidade em todos os centros de investigação do ELSA-Brasil 23. Os exames de CT, HDL-C, LDL-C e TG foram coletados em jejum de 12 horas. CT e TG foram mensurados pelo método enzimático colorimétrico; HDL-C e LDL-C (quando TG > 400mg/dL) pelo método colorimétrico homogêneo sem precipitação (ADVIA 1200, Siemens). Para o LDL-C, quando TG $\leq 400 \mathrm{mg} / \mathrm{dL}$, foi utilizada a Equação de Friedewald 24. Todos os valores foram expressos em mg/dL.

Foi calculada a razão TG/HDL-C e considerados como maiores riscos cardiovasculares valores $>$ 3,5 para homens e > 2,5 para mulheres 25. TG e HDL-C também foram analisados separadamente, sendo considerados indivíduos com hipertrigliceridemia aqueles com valores de $\mathrm{TG} \geq 150 \mathrm{mg} / \mathrm{dL}$, e baixo valor de HDL-C $<40 \mathrm{mg} / \mathrm{dL}$ para homens e $<50 \mathrm{mg} / \mathrm{dL}$ para mulheres. Para os valores de LDL-C foram considerados maiores riscos valores $\geq 160 \mathrm{mg} / \mathrm{dL} 4$.

A dosagem da PCR pelo método ultrassensível (PCR-us) no sangue foi realizada por nefelometria (BN II; Siemens) e os resultados foram expressos em mg/L. Consideraram-se níveis altos de PCR valores $\geq 3 \mathrm{mg} / \mathrm{L} 26$.

\section{Covariáveis}

Foram incluídas nos modelos de análise as variáveis sociodemográficas (idade, raça/cor e renda per capita) e de estilo de vida (consumo de álcool, prática de atividade física no lazer e hábito de fumar). O consumo de álcool foi avaliado em gramas de etanol/semana. A atividade física no lazer foi mensurada pelo Questionário Internacional de Atividade Física (IPAQ), versão longa, e o padrão de atividade classificada de acordo com a recomendação do IPAQ como caminhada, atividades físicas de intensi- 
dades moderada e vigorosa 27. A variável foi categorizada posteriormente em fraca, moderada e forte. O hábito de fumar foi categorizado em nunca fumou, ex-fumante e fumante atual. Também foram incluídos nos modelos a presença de obesidade abdominal (valores de circunferência de cintura $\geq$ $80 \mathrm{~cm}$ para mulheres e $\geq 94 \mathrm{~cm}$ para homens) e o status menopausal em mulheres ( $\mathrm{sim} / \mathrm{não}$ ). A circunferência da cintura foi aferida utilizando-se fita métrica inelástica, em torno do ponto médio entre a última costela e a crista ilíaca.

Variáveis dietéticas também foram consideradas possíveis confundidores e incluídas nas análises, como energia total (kcal) e grupos alimentares não lácteos (frutas, verduras e legumes, cereais integrais, peixe, carnes não processadas e carnes processadas; expressos em g/dia), estimados com base no QFA.

\section{Análise estatística}

Os dados foram analisados com o software IBM SPSS, versão 22 (https://www.ibm.com/). Todas as análises foram estratificadas por sexo. Variáveis contínuas foram expressas em média e desvio padrão, e as categóricas expressas em frequência e percentual. As características dos participantes foram comparadas entre os sexos utilizando-se teste t de Student (variáveis contínuas) e teste do qui-quadrado (variáveis categóricas).

A análise bivariada foi realizada por meio de regressão logística. As variáveis PCR, LDL-C, TG, HDL-C e razão TG/LDL alterados foram consideradas variáveis dependentes, e a ingestão diária de lácteos totais, em quatro categorias ( $\leq 1,>1-2,>2-4$ e $>4$ porções/dia), como variável explicativa. As associações foram estimadas por meio do odds ratios (OR) ajustado pela idade, utilizando-se o grupo com o menor consumo de lácteos ( $\leq 1$ porção/dia) como referência. Os modelos ainda foram ajustados para potenciais fatores de confusão: raça/cor, renda per capita, consumo de álcool, nível de atividade física, hábito de fumar, obesidade abdominal, status menopausal (em mulheres), energia total e consumo de grupos alimentares não lácteos. A OR com intervalo de 95\% de confiança (IC95\%) foi usada como medida de efeito. Para as análises, um nível de significância de 5\% foi adotado.

Também foram testadas as associações entre diferentes subgrupos: lácteos integrais, com reduzido teor de gordura, fermentados, leite, queijo, iogurte e manteiga (em porção/dia) e valores de PCR, TG/ HDL-C, LDL-C, TG, HDL-C, por meio de modelos de regressão linear multivariada. Devido à forte assimetria das variáveis dependentes TG, TG/HDL-C e PCR, as mesmas foram log transformadas para as análises. Para as análises da variável dependente PCR, os participantes com valores abaixo do limite de detecção do exame $(0,175 \mathrm{mg} / \mathrm{L})(\mathrm{n}=283)$ foram excluídos dos modelos de regressão linear.

\section{Aspectos éticos}

O ELSA-Brasil foi aprovado pela Comissão Nacional de Ética em Pesquisa (CONEP - 976/2006) e pelos comitês de ética em pesquisa de cada instituição. Todos os participantes assinaram o Termo de Consentimento Livre Esclarecido.

\section{Resultados}

As características sociodemográficas, de saúde e dietéticas dos participantes estão descritas na Tabela 1. A população era de maioria branca (53,7\%), com média de idades de 49,98 $\pm 8,48$ anos e índice de massa corporal (IMC) de $26,19 \mathrm{~kg} / \mathrm{m}^{2}( \pm 4,33)$. Os homens apresentaram maior consumo de álcool, carnes e peixes, e menor consumo de frutas, verduras e legumes e grãos integrais, em relação às mulheres. Ainda, os homens registraram maiores valores médios TG e LDL-C, maior nível de atividade física e hábito de fumar; e menores valores de renda per capita, PCR, HDL-C e obesidade abdominal.

Em relação ao consumo, a média de lácteos totais foi de 3,09 $\pm 2,19$ porções/dia, sendo 3,22 $\pm 2,15$ porções/dia nas mulheres e 2,92 $\pm 2,23$ porções/dia nos homens. As mulheres apresentaram maior consumo de lácteos com reduzido teor de gordura $(+0,50 \pm 0,03$ porção/dia, $\mathrm{p}<0,001)$ e fermentados $(+0,26 \pm 0,03$ porção/dia, $\mathrm{p}<0,001)$; e menor consumo de lácteos integrais $(-0,14 \pm 0,03$ porção/dia, $\mathrm{p}<0,001)$ em relação aos homens. Nos subgrupos de lácteos com reduzido teor de gordura e lácteos 
Tabela 1

Características dos participantes por sexo. Estudo Longitudinal de Saúde do Adulto (ELSA-Brasil), 2008-2010.

\begin{tabular}{|c|c|c|c|c|}
\hline Características & $\begin{array}{c}\text { Homens } \\
(n=4.031)\end{array}$ & $\begin{array}{l}\text { Mulheres } \\
(n=5.341)\end{array}$ & Valor de $p$ * & $\begin{array}{c}\text { Total } \\
(\mathrm{n}=9.372)\end{array}$ \\
\hline Idade (anos) [média \pm DP] & $49,89 \pm 8,69$ & $50,05 \pm 8,31$ & 0,369 & $49,98 \pm 8,48$ \\
\hline Renda per capita $(\mathrm{R} \$)$ [média $\pm \mathrm{DP}]$ & $1.663,64 \pm 1.331,72$ & $1.805,23 \pm 1.447,90$ & $<0,001$ & $1.744,34 \pm 1.400,80$ \\
\hline \multicolumn{5}{|l|}{ Raça $[\mathrm{n}(\%)]$} \\
\hline Brancos & $2.154(53,4)$ & $2.869(53,7)$ & 0,787 & $5.023(53,7)$ \\
\hline Não brancos & $1.877(46,6)$ & $2.472(46,3)$ & & $4.349(46,4)$ \\
\hline \multicolumn{5}{|l|}{ Hábito de fumar [n (\%)] } \\
\hline Nunca fumou & $2.249(55,8)$ & $2.439(64,4)$ & $<0,001$ & $5.688(60,7)$ \\
\hline Ex-fumante & $1.223(30,3)$ & $1.252(23,4)$ & & $2.475(26,4)$ \\
\hline Fumante atual & $559(13,9)$ & $650(12,2)$ & & $1.209(12,9)$ \\
\hline Menopausa [n (\%)] & - & $1.897(35,5)$ & & \\
\hline Obesidade abdominal [n (\%)] & $1.772(44,0)$ & $3.463(64,8)$ & $<0,001$ & $5.235(55,9)$ \\
\hline Ingestão de álcool (g etanol/semana) [média × DP] & $85,78 \pm 131,78$ & $24,74 \pm 51,98$ & $<0,001$ & $51,01 \pm 99,61$ \\
\hline \multicolumn{5}{|l|}{ Atividade Física [média \pm DP] } \\
\hline Fraca & $2.956(55,8)$ & $4.252(79,7)$ & $<0,001$ & $7.211(76,9)$ \\
\hline Moderada & $577(30,3)$ & $664(12,4)$ & & $1.241(13,2)$ \\
\hline Forte & $498(13,9)$ & $422(7,9)$ & & $920(9,8)$ \\
\hline Proteína C-reativa (mg/L) [média \pm DP] & $1,79 \pm 1,80$ & $2,29 \pm 2,18$ & $<0,001$ & $2,08 \pm 2,04$ \\
\hline Triglicerídeos (mg/dL) [média \pm DP] & $149,80 \pm 102,82$ & $110,59 \pm 63,39$ & $<0,001$ & $127,45 \pm 84,93$ \\
\hline LDL-C (mg/dL) [média \pm DP] & $134,76 \pm 34,62$ & $132,93 \pm 33,55$ & 0,010 & $133,72 \pm 34,03$ \\
\hline HDL-C (mg/dL) [média \pm DP] & $51,49 \pm 12,38$ & $62,65 \pm 14,54$ & $<0,001$ & $57,85 \pm 14,73$ \\
\hline \multicolumn{5}{|l|}{ Grupos alimentares (g/dia) [média \pm DP] } \\
\hline Frutas & $489,99 \pm 404,65$ & $543,28 \pm 405,89$ & $<0,001$ & $520,36 \pm 406,19$ \\
\hline Verduras e legumes & $200,13 \pm 141,01$ & $219,20 \pm 146,74$ & $<0,001$ & $211,00 \pm 144,61$ \\
\hline Carne não processada & $181,30 \pm 124,50$ & $151,01 \pm 106,39$ & $<0,001$ & $164,04 \pm 115,50$ \\
\hline Carne processada & $24,65 \pm 25,85$ & $19,23 \pm 21,75$ & $<0,001$ & $21,57 \pm 23,75$ \\
\hline Peixe & $52,99 \pm 60,69$ & $44,75 \pm 55,24$ & $<0,001$ & $48,29 \pm 57,79$ \\
\hline Grãos integrais & $40,14 \pm 77,21$ & $44,14 \pm 66,08$ & $<0,001$ & $42,42 \pm 71,11$ \\
\hline
\end{tabular}

DP: desvio padrão.

* Qui-quadrado para variáveis categóricas; teste t de Student para variáveis quantitativas.

fermentados, o queijo foi o alimento com o maior consumo, tanto em homens quanto em mulheres, em todas as categorias de consumo (Tabela 2).

Participantes nas categorias de maior ingestão de lácteos totais (> 2-4 e > 4 porções/dia) registraram menor chance de apresentar valores aumentados da razão TG/HDL-C, mas não de LDL-C e PCR. Os menores valores de OR para TG/HDL-C no modelo multivariado (0,70; IC95\%: 0,55-0,90 em homens e 0,55; IC95\%: 0,43-0,70 em mulheres) foram encontrados no grupo com consumo > 4 porções/dia de lácteos. Analisando-se os parâmetros TG e HDL-C separadamente, em homens e mulheres, um maior consumo de lácteos totais esteve associado à menor chance de hipertrigliceridemia (OR: 0,66; IC95\%: 0,53-0,83 em homens; OR: 0,55; IC95\%: 0,43-0,71 em mulheres), e somente nas mulheres um maior consumo de lácteos foi associado a uma menor chance de apresentar baixos valores de HDL-C (OR: 0,68; IC95\%: 0,53-0,89) (Tabela 3).

As Tabelas 4 e 5 mostram os resultados das análises de regressão linear múltipla. Nos homens, os lácteos integrais, fermentados, iogurte, queijo e manteiga foram inversamente associados aos valores de $\log$ TG/HDL-C e $\log$ TG (Tabela 4). Nas mulheres, lácteos integrais, fermentados, queijo e manteiga foram inversamente associados aos valores $\log \mathrm{TG} / \mathrm{HDL}-\mathrm{C}, \log \mathrm{TG}$ e positivamente associados ao HDL-C (Tabela 5). Lácteos com reduzido teor de gordura foram inversamente associados apenas 
Tabela 2

Consumo dos diferentes subgrupos de produtos lácteos (porções/dia), segundo sexo. Estudo Longitudinal de Saúde do Adulto (ELSA-Brasil), $2008-2010$.

\begin{tabular}{|c|c|c|c|c|}
\hline \multirow[t]{2}{*}{ Subgrupos de lácteos } & \multicolumn{4}{|c|}{ Consumo de lácteos totais (porções/dia) } \\
\hline & Homens (médias \pm DP) & Mulheres (médias \pm DP) & Valor de $p$ * & Total (médias \pm DP) \\
\hline Lácteos com reduzido teor de gordura (medianas) & 0,47 & 1,07 & & \\
\hline Leite desnatado e semidesnatado & $0,36 \pm 0,81$ & $0,55 \pm 0,93$ & $<0,001$ & $0,47 \pm 0,88$ \\
\hline logurte light & $0,06 \pm 0,24$ & $0,16 \pm 0,37$ & $<0,001$ & $0,12 \pm 0,32$ \\
\hline Queijo com reduzido teor de gordura & $0,71 \pm 1,12$ & $0,92 \pm 1,22$ & $<0,001$ & $0,83 \pm 1,18$ \\
\hline Lácteos integrais (medianas) & 1,00 & 0,87 & & \\
\hline Leite integral & $0,56 \pm 0,97$ & $0,47 \pm 0,90$ & $<0,001$ & $0,51 \pm 0,93$ \\
\hline logurte integral & $0,18 \pm 0,38$ & $0,21 \pm 0,41$ & $<0,001$ & $0,20 \pm 0,40$ \\
\hline Queijos com teor regular de gordura & $0,69 \pm 0,69$ & $0,60 \pm 0,88$ & $<0,001$ & $0,64 \pm 0,93$ \\
\hline Lácteos fermentados (medianas) & 1,14 & 1,47 & & \\
\hline logurte total & $0,24 \pm 0,42$ & $0,38 \pm 0,49$ & $<0,001$ & $0,32 \pm 0,46$ \\
\hline Queijo total & $1,39 \pm 1,55$ & $1,52 \pm 1,50$ & $<0,001$ & $1,46 \pm 1,52$ \\
\hline Manteiga & $0,37 \pm 0,75$ & $0,30 \pm 0,66$ & $<0,001$ & $0,33 \pm 0,70$ \\
\hline
\end{tabular}

* Teste t de Student.

\section{Tabela 3}

Odds ratios (OR) ajustados para associação entre consumo de lácteos e marcadores de risco cardiovascular em homens e mulheres. Estudo Longitudinal de Saúde do Adulto (ELSA-Brasil), 2008-2010.

\begin{tabular}{|c|c|c|c|c|}
\hline & \multicolumn{4}{|c|}{ Lácteos totais (porções/dia) [OR (IC95\%)] } \\
\hline & $\leq 1$ & $>1-2$ & $>2-4$ & $>4$ \\
\hline \multicolumn{5}{|l|}{ Homens $(n=4.031)$} \\
\hline \multicolumn{5}{|l|}{$\mathrm{PCR}(\geq 3 \mathrm{mg} / \mathrm{dL})$} \\
\hline Modelo ajustado para idade & Referência & $0,93(0,72-1,19)$ & $0,77(0,61-0,97)$ & $0,89(0,69-1,13)$ \\
\hline Valor de $\mathrm{p}$ & & 0,545 & 0,026 & 0,339 \\
\hline Modelo multivariado * & Referência & $0,99(0,77-1,29)$ & $0,86(0,67-1,11)$ & $0,95(0,72-1,23)$ \\
\hline Valor de $p$ & & 0,956 & 0,257 & 0,738 \\
\hline \multicolumn{5}{|l|}{ TG/HDL-C $(>3,5)$} \\
\hline Modelo ajustado para idade & Referência & $0,90(0,73-1,01)$ & $0,76(0,63-0,92)$ & $0,74(0,60-0,90)$ \\
\hline Valor de $p$ & & 0,291 & 0,005 & 0,004 \\
\hline Modelo multivariado * & Referência & $0,88(0,71-1,09)$ & $0,75(0,61-0,93)$ & $0,70(0,55-0,90)$ \\
\hline Valor de $\mathrm{p}$ & & 0,252 & 0,007 & 0,004 \\
\hline \multicolumn{5}{|l|}{ LDL-C ( $\geq 160$ mg/dL) } \\
\hline Modelo ajustado para idade & Referência & $0,92(0,72-1,17)$ & $1,03(0,83-1,29)$ & $1,02(0,81-1,29)$ \\
\hline Valor de $p$ & & 0,514 & 0,766 & 0,862 \\
\hline Modelo multivariado * & Referência & $0,92(0,72-1,17)$ & $1,04(0,83-1,32)$ & $1,06(0,81-1,38)$ \\
\hline Valor de $\mathrm{p}$ & & 0,502 & 0,714 & 0,665 \\
\hline \multicolumn{5}{|l|}{ TG ( $\geq 150$ mg/dL) } \\
\hline Modelo ajustado para idade & Referência & $0,86(0,70-1,05)$ & $0,79(0,66-0,95)$ & $0,70(0,58-0,86)$ \\
\hline Valor de $\mathrm{p}$ & & 0,131 & 0,012 & 0,001 \\
\hline Modelo multivariado * & Referência & $0,83(0,68-1,03)$ & $0,78(0,64-0,95)$ & $0,66(0,53-0,83)$ \\
\hline Valor de $\mathrm{p}$ & & 0,086 & 0,015 & $<0,001$ \\
\hline
\end{tabular}

(continua) 
Lácteos totais (porções/dia) [OR (IC95\%)]

$\leq 1$

$>1-2$

$>2-4$

$>4$

\section{Homens $(n=4.031)$}

HDL-C $(<40 \mathrm{mg} / \mathrm{dL})$

Modelo ajustado para idade

valor de $\mathrm{p}$

Modelo multivariado *

Valor de $\mathrm{p}$

Mulheres $(\mathbf{n}=\mathbf{5 . 3 4 1})$

PCR ( $\geq 3 \mathrm{mg} / \mathrm{dL}$ )

Modelo ajustado para idade

Valor de $\mathrm{p}$

Modelo multivariado *

Valor de $\mathrm{p}$

TG/HDL-C $(>2,5)$

Modelo ajustado para idade

Valor de $p$

Modelo multivariado *

Valor de $p$

LDL-C ( $\geq 160 \mathrm{mg} / \mathrm{dL}$ )

Modelo ajustado para idade

Valor de $\mathrm{p}$

Modelo multivariado *

Valor de $\mathrm{p}$

TG ( $\geq 150 \mathrm{mg} / \mathrm{dL})$

Modelo ajustado para idade

valor de $\mathrm{p}$

Modelo multivariado *

Valor de $\mathrm{p}$

HDL-C (<50mg/dL)

Modelo ajustado para idade

Valor de $p$

Modelo multivariado *

Valor de $\mathrm{p}$

\begin{tabular}{|c|c|}
\hline \multirow[t]{2}{*}{ Referência } & $1,20(0,91-1,60)$ \\
\hline & 0,201 \\
\hline \multirow[t]{2}{*}{ Referência } & $1,24(0,93-1,63)$ \\
\hline & 0,149 \\
\hline \multirow[t]{2}{*}{ Referência } & $1,00(0,81-1,25)$ \\
\hline & 0,959 \\
\hline \multirow[t]{2}{*}{ Referência } & $1,05(0,84-1,31)$ \\
\hline & 0,680 \\
\hline \multirow[t]{2}{*}{ Referência } & $0,77(0,62-0,96)$ \\
\hline & 0,022 \\
\hline \multirow[t]{2}{*}{ Referência } & $0,80(0,63-1,01)$ \\
\hline & 0,064 \\
\hline \multirow[t]{2}{*}{ Referência } & $1,04(0,82-1,33)$ \\
\hline & 0,733 \\
\hline \multirow[t]{2}{*}{ Referência } & $1,09(0,85-1,39)$ \\
\hline & 0,501 \\
\hline \multirow[t]{2}{*}{ Referência } & $0,78(0,61-0,99)$ \\
\hline & 0,040 \\
\hline \multirow[t]{2}{*}{ Referência } & $0,80(0,62-1,02)$ \\
\hline & 0,067 \\
\hline \multirow[t]{2}{*}{ Referência } & $0,83(0,65-1,06)$ \\
\hline & 0,135 \\
\hline \multirow[t]{2}{*}{ Referência } & $0,86(0,67-1,11)$ \\
\hline & 0,243 \\
\hline
\end{tabular}

$$
\begin{gathered}
1,04(0,79-1,35) \\
0,792 \\
1,07(0,81-1,43) \\
0,617
\end{gathered}
$$

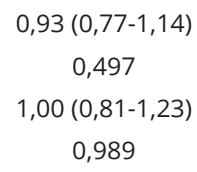

0,356

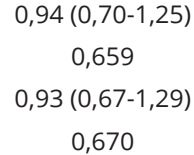

0,004

IC95\%: intervalo de 95\% de confiança; PCR: proteína C-reativa; TG: triglicerídeo.

* Ajustado por idade, renda, raça, status menopausal, obesidade abdominal, tabagismo, ingestão de álcool, atividade física, calorias totais, grupos alimentares não lácteos.

com $\log \mathrm{TG} / \mathrm{HDL}-\mathrm{C}$, em homens e mulheres, e com $\log \mathrm{TG}$ em mulheres. O único lácteo que mostrou associação com $\log \mathrm{PCR}$ foi a manteiga, somente em mulheres.

Em análises de sensibilidade, foi investigado se a associação entre os grupos de lácteos integrais e com reduzido teor de gordura com o $\log$ TG/HDL-C estava sendo impulsionada pelos lácteos fermentados. Analisando, dentro de cada grupo, leite e fermentados (iogurte e queijo) separadamente, as associações permaneceram para os fermentados integrais $(-0,009 \pm 0,004 ; p=0,017$ em homens e - $-0,013 \pm 0,003 ; p<0,001$ nas mulheres $)$ e com baixo teor de gordura $(-0,008 \pm 0,004 ; p=0,029$ em homens e $-0,008 \pm 0,003 ; p=0,001$ nas mulheres), enquanto nenhum tipo de leite mostrou associação com os valores de $\log$ TG/HDL-C. Dentre os homens, analisando somente os fermentados, excluindo o leite, o grupo com reduzido teor de gordura passou a apresentar uma relação inversa e significativa também com logTG $(-0,006 \pm 0,003 ; \mathrm{p}=0,041)$. 
Tabela 4

Associação de diferentes subgrupos de lácteos com $\log \mathrm{PCR}$, $\log \mathrm{TG} / \mathrm{HDL}-\mathrm{C}, \log \mathrm{TG}, \mathrm{HDL}-\mathrm{C}$ e LDL-C nas análises de regressão linear múltipla em homens. Estudo Longitudinal de Saúde do Adulto (ELSA-Brasil), 2008-2010.

\begin{tabular}{|c|c|c|c|c|c|}
\hline Subgrupos de lácteos (porção/dia) & $\log \mathrm{PCR}$ & $\operatorname{logTG/HDL-C}$ & $\log \mathrm{TG}$ & HDL-C & LDL-C \\
\hline \multicolumn{6}{|l|}{ Lácteos com reduzido teor de gordura } \\
\hline$B \pm E P$ & $0,000 \pm 0,004$ & $-0,006 \pm 0,003$ & $-0,004 \pm 0,002$ & $0,224 \pm 0,129$ & $0,054 \pm 0,380$ \\
\hline Valor de $\mathrm{p}$ & 0,968 & 0,030 & 0,067 & 0,083 & 0,885 \\
\hline \multicolumn{6}{|l|}{ Lácteos integrais } \\
\hline$B \pm E P$ & $-0,003 \pm 0,004$ & $-0,008 \pm 0,003$ & $-0,008 \pm 0,002$ & $-0,021 \pm 0,134$ & $-0,149 \pm 0,396$ \\
\hline Valor de $\mathrm{p}$ & 0,470 & 0,010 & 0,002 & 0,876 & 0,707 \\
\hline \multicolumn{6}{|l|}{ Lácteos fermentados } \\
\hline$B \pm E P$ & $-0,002 \pm 0,004$ & $-0,009 \pm 0,003$ & $-0,008 \pm 0,002$ & $0,164 \pm 0,123$ & $0,168 \pm 0,362$ \\
\hline Valor de $\mathrm{p}$ & 0,635 & 0,001 & 0,001 & 0,184 & 0,642 \\
\hline \multicolumn{6}{|l|}{ Leite } \\
\hline$B \pm E P$ & $-0,001 \pm 0,006$ & $-0,007 \pm 0,004$ & $-0,006 \pm 0,003$ & $0,048 \pm 0,180$ & $-0,521 \pm 0,531$ \\
\hline Valor de $\mathrm{p}$ & 0,828 & 0,088 & 0,073 & 0,792 & 0,326 \\
\hline \multicolumn{6}{|l|}{ logurte } \\
\hline$B \pm E P$ & $0,000 \pm 0,015$ & $-0,020 \pm 0,010$ & $-0,020 \pm 0,008$ & $-0,083 \pm 0,454$ & $-1,632 \pm 1,337$ \\
\hline Valor de $\mathrm{p}$ & 0,996 & 0,046 & 0,016 & 0,854 & 0,222 \\
\hline \multicolumn{6}{|l|}{ Queijo } \\
\hline $\mathrm{B} \pm \mathrm{EP}$ & $-0,002 \pm 0,004$ & $-0,008 \pm 0,003$ & $-0,007 \pm 0,002$ & $0,191 \pm 0,130$ & $0,323 \pm 0,384$ \\
\hline Valor de $p$ & 0,614 & 0,004 & 0,004 & 0,144 & 0,400 \\
\hline \multicolumn{6}{|l|}{ Manteiga } \\
\hline $\mathrm{B} \pm \mathrm{EP}$ & $-0,003 \pm 0,008$ & $-0,016 \pm 0,006$ & $-0,012 \pm 0,005$ & $0,424 \pm 0,250$ & $1,141 \pm 0,738$ \\
\hline Valor de $p$ & 0,655 & 0,005 & 0,011 & 0,091 & 0,122 \\
\hline
\end{tabular}

B: coeficiente $\beta$; EP: erro padrão; PCR: proteína C-reativa; TG: triglicerídeo.

Nota 1: valores apresentados como coeficiente $\beta \pm$ erro padrão $(B \pm E P)$ utilizando regressão linear ajustada por idade, raça, obesidade abdominal, tabagismo, ingestão de álcool, atividade física, calorias totais, grupos alimentares não lácteos.

Nota 2: TG, LDL-C e HDL-C expressos em mg/dL, PCR expresso em mg/L. Subgrupos de lácteos expressos em porção/dia.

Nas mulheres, a associação positiva entre lácteos fermentados e queijo com HDL-C mostrou diferenças de acordo com o conteúdo de gordura destes produtos, uma vez que, analisando separadamente fermentados e queijo integrais e com reduzido teor de gordura, a associação permaneceu significativa somente para fermentados integrais $(0,764 \pm 0,199 ; \mathrm{p}<0,001)$ e queijo integral $(1,263$ $\pm 0,250 ; \mathrm{p}<0,001)$, não sendo observado o mesmo para os produtos com reduzido teor de gordura. Analisado isoladamente, iogurte integral não mostrou associação com o HDL-C.

\section{Discussão}

Neste trabalho, os produtos lácteos foram associados, em homens e mulheres, a uma menor chance de apresentar valores aumentados de razão TG/HDL-C, um preditor importante de doenças cardiovasculares. Indivíduos com consumo estimado maior que 4 porções/dia de lácteos totais registraram menor chance de apresentar valores alterados da razão TG/HDL-C do que aqueles classificados no menor grupo de ingestão. Esses resultados são apoiados pelas associações inversas encontradas entre diferentes subgrupos de lácteos e a razão TG/HDL-C na amostra estudada, o que corrobora evidências epidemiológicas recentes que sugerem uma associação inversa entre a ingestão de produtos lácteos, particularmente laticínios fermentados, e DCV 28. Por outro lado, não foi encontrada associação entre o consumo de produtos lácteos e seus subgrupos com valores de LDL-C e de PCR, utilizada como marcador inflamatório sistêmico. 
Tabela 5

Associação de diferentes subgrupos de lácteos com $\log \mathrm{PCR}, \log \mathrm{TG} / \mathrm{HDL}-\mathrm{C}, \log \mathrm{TG}, \mathrm{HDL}-\mathrm{C}$ e LDL-C nas análises de regressão linear múltipla em mulheres. Estudo Longitudinal de Saúde do Adulto (ELSA-Brasil), 2008-2010.

\begin{tabular}{|c|c|c|c|c|c|}
\hline Subgrupos de lácteos (porção/dia) & $\log \mathrm{PCR}$ & $\operatorname{logTG/HDL-C~}$ & $\log \mathrm{TG}$ & HDL-C & LDL-C \\
\hline \multicolumn{6}{|l|}{ Lácteos com reduzido teor de gordura } \\
\hline$B \pm E P$ & $0,004 \pm 0,003$ & $-0,005 \pm 0,002$ & $-0,004 \pm 0,002$ & $0,208 \pm 0,116$ & $0,056 \pm 0,269$ \\
\hline Valor de $\mathrm{p}$ & 0,211 & 0,005 & 0,011 & 0,073 & 0,834 \\
\hline \multicolumn{6}{|l|}{ Lácteos integrais } \\
\hline$B \pm E P$ & $-0,003 \pm 0,004$ & $-0,009 \pm 0,002$ & $-0,007 \pm 0,002$ & $0,381 \pm 0,146$ & $-0,377 \pm 0,341$ \\
\hline Valor de $\mathrm{p}$ & 0,469 & $<0,001$ & 0,001 & 0,009 & 0,268 \\
\hline \multicolumn{6}{|l|}{ Lácteos fermentados } \\
\hline$B \pm E P$ & $0,001 \pm 0,004$ & $-0,011 \pm 0,002$ & $-0,008 \pm 0,002$ & $0,472 \pm 0,126$ & $-0,024 \pm 0,294$ \\
\hline Valor de $p$ & 0,866 & $<0,001$ & $<0,001$ & $<0,001$ & 0,944 \\
\hline \multicolumn{6}{|l|}{ Leite } \\
\hline $\mathrm{B} \pm \mathrm{EP}$ & $0,004 \pm 0,005$ & $-0,005 \pm 0,003$ & $-0,004 \pm 0,002$ & $0,123 \pm 0,184$ & $-0,412 \pm 0,429$ \\
\hline Valor de $\mathrm{p}$ & 0,404 & 0,088 & 0,073 & 0,505 & 0,337 \\
\hline \multicolumn{6}{|l|}{ logurte } \\
\hline$B \pm E P$ & $0,022 \pm 0,011$ & $-0,002 \pm 0,007$ & $-0,000 \pm 0,005$ & $0,287 \pm 0,398$ & $0,261 \pm 0,924$ \\
\hline Valor de $\mathrm{p}$ & 0,054 & 0,782 & 0,954 & 0,470 & 0,778 \\
\hline \multicolumn{6}{|l|}{ Queijo } \\
\hline $\mathrm{B} \pm \mathrm{EP}$ & $-0,002 \pm 0,004$ & $-0,013 \pm 0,002$ & $-0,009 \pm 0,002$ & $0,515 \pm 0,136$ & $-0,054 \pm 0,316$ \\
\hline Valor de $p$ & 0,634 & $<0,001$ & $<0,001$ & $<0,001$ & 0,864 \\
\hline \multicolumn{6}{|l|}{ Manteiga } \\
\hline $\mathrm{B} \pm \mathrm{EP}$ & $-0,019 \pm 0,008$ & $-0,018 \pm 0,005$ & $-0,012 \pm 0,004$ & $0,969 \pm 0,291$ & $1,310 \pm 0,677$ \\
\hline Valor de $p$ & 0,024 & $<0,001$ & 0,004 & 0,001 & 0,053 \\
\hline
\end{tabular}

B: coeficiente $\beta$; EP: erro padrão; PCR: proteína C-reativa; TG: triglicerídeo.

Nota 1: valores apresentados como coeficiente $\beta \pm$ erro padrão $(B \pm E P)$ utilizando regressão linear ajustada por idade, raça, status menopausal, obesidade abdominal, tabagismo, ingestão de álcool, atividade física, calorias totais, grupos alimentares não lácteos.

Nota 2: TG, LDL-C e HDL-C expressos em mg/dL, PCR expresso em mg/L. Subgrupos de lácteos expressos em porção/dia.

Os laticínios fermentados, independentemente do conteúdo de gordura, foram inversamente associados aos valores de $\log$ TG/HDL-C e $\log$ TG em homens e mulheres. No entanto, analisando iogurte e queijo separadamente, houve diferenças entre os sexos. Enquanto o queijo foi inversamente associado a $\log$ TG/HDL-C e $\log$ TG em ambos os sexos, a associação com iogurte só foi significativa para os homens. Uma meta-análise dose-resposta recente, que avaliou a relação entre a ingestão de produtos lácteos fermentados e o risco de desenvolvimento de DCV, encontrou uma relação inversa entre queijo (10g/dia) e DCV (RR: 0,98; IC95\%: 0,95-1,00), e nenhuma associação significativa foi encontrada para o iogurte 29 . Um estudo transversal com 6.572 homens e mulheres com sobrepeso/ obesidade e síndrome metabólica encontrou que o maior consumo de queijo foi inversamente associado à prevalência de hipertrigliceridemia, e não houve associação para os diferentes tipos de iogurte (integral e reduzido teor de gordura) 30. Por outro lado, um estudo longitudinal conduzido com indivíduos com alto risco cardiovascular encontrou uma associação entre iogurte com baixo teor de gordura e hipertrigliceridemia, não sendo encontradas associações com queijo 31 .

Apesar da divergência de evidências na literatura, os resultados deste estudo corroboram outros que mostraram uma associação inversa entre o consumo de queijo e valores de TG. Análise transversal dos dados do Oslo Health Study 32, que examinou diferentes componentes da síndrome metabólica, encontrou uma associação inversa entre o consumo de queijo e TG em 18.770 homens e mulheres adultos da Noruega. Um estudo longitudinal francês 33, com 3.417 adultos de ambos os sexos, mostrou uma associação entre maior consumo de queijo e menores aumentos nos valores de TG, após nove anos de acompanhamento. Um ensaio clínico randomizado que avaliou o efeito de diferentes 
tipos de queijo no perfil lipídico após oito semanas de intervenção, encontrou uma associação entre consumo de queijo com alto teor de gordura $(27 \%)$ e redução significativa nos valores de TG em indivíduos com síndrome metabólica 13.

Em relação ao HDL-C, um efeito significativo dos produtos lácteos foi encontrado somente nas mulheres. O maior consumo de produtos lácteos fermentados, sobretudo queijo, foi relacionado a maiores valores de HDL-C nesse grupo, sendo que o conteúdo de gordura nesses alimentos pareceu influenciar essa relação. Høstmark \& Tomten 32 também encontraram uma associação positiva entre o consumo de queijo e valores de HDL-C, no entanto, não foram realizadas análises considerando o conteúdo de gordura do alimento e nem as diferenças entre os sexos. Um ensaio clínico randomizado com 85 homens e mulheres, que comparou três tipos de dieta: com queijo integral, queijo com reduzido teor de gordura e dieta sem queijo (substituído por carboidrato) encontrou um efeito do queijo integral, em comparação à dieta sem queijo, no aumento da HDL-C apenas em mulheres ${ }^{34}$. Em um modelo animal, Thorning et al. 35 encontraram que o consumo de queijo integral foi relacionado a um aumento nas concentrações de HDL-C, sendo que o mesmo não foi observado para o queijo com reduzido teor de gordura.

Os mecanismos pelos quais os lácteos fermentados teriam efeito benéfico na saúde cardiovascular não são totalmente esclarecidos. Acredita-se que a matriz alimentar dos lácteos poderia modificar o perfil lipídico devido a um efeito sinérgico entre diferentes componentes e bactérias probióticas. A alta concentração de cálcio no queijo poderia inibir a absorção de ácidos graxos, aumentando sua excreção fecal 36. Outro mecanismo possível se refere às diferentes cepas de bactérias presentes nos lácteos fermentados, que poderiam modular a microbiota intestinal, favorecendo a produção de ácidos graxos de cadeia curta que atuam no metabolismo lipídico ${ }^{37}$. Um possível efeito da membrana dos glóbulos de gordura do leite também tem sido investigado, podendo estar associado à menor absorção intestinal de LDL-C e à regulação da expressão de genes relacionados ao metabolismo lipídico 38 . Ainda, em um modelo experimental com ratos, Higurashi et al. 39 encontraram relação entre consumo de queijo e maiores valores de adiponectina, sugerindo um possível efeito dos peptídeos presentes no alimento na sinalização deste hormônio, que poderia estar relacionado aos efeitos benéficos ligados ao consumo de queijo.

Nenhum dos produtos lácteos estudados mostrou associação significativa com o LDL-C, apesar de a manteiga ter mostrado uma associação positiva muito próxima ao limite de significância $(\mathrm{p}=0,053)$ nas mulheres. Associação positiva também foi encontrada para manteiga e HDL-C nesse grupo. Ensaios clínicos têm mostrado uma maior associação entre ácidos graxos saturados (AGS) da manteiga e aumento de LDL-C, em comparação aos AGS do queijo 40 e a outros tipos de gordura 41 . Apesar disso, estudos também têm relacionado o consumo de manteiga a maiores valores de HDL-C 41 e maior capacidade de efluxo de colesterol mediada pelo HDL-C, em comparação a uma dieta com AGS do queijo ou uma dieta com elevada quantidade de carboidrato 42. Uma meta-análise de estudos prospectivos não encontrou associação significativa entre o consumo de manteiga com DCV, doença coronariana ou infarto, e uma associação inversa foi demonstrada para o diabetes. Os autores sugerem que, apesar dos efeitos adversos de algumas gorduras lácteas sobre fatores de risco como LDL-C, a presença potencial de outros mecanismos benéficos da manteiga poderia compensar pelo menos parcialmente estes efeitos 43 .

Neste trabalho, um resultado não esperado foi a associação significativamente inversa entre consumo de manteiga e os valores de $\log \mathrm{TG}$ (homens e mulheres) e $\log \mathrm{PCR}$ (mulheres). Mozaffarian et al. 44 investigaram a relação entre ácido trans-palmitoleico (trans-16:1n-7), um biomarcador do consumo de gordura láctea, e diferentes fatores de risco metabólico em um estudo prospectivo com 3.736 adultos do Cardiovascular Health Study. Os autores encontraram, além de uma associação positiva com HDL-C, uma associação inversa com TG $(-19 \%$; $<$ < 0,001) e PCR $(-13,8 \%$; p = 0,050) comparando o quintil de maior concentração com o de menor concentração plasmática de trans-16:1n-7. O maior nível de trans-16:1n-7 também foi associado à menor incidência de diabetes mellitus. Esses achados corroboram os resultados do presente estudo e as análises prévias na população do ELSA-Brasil, que mostraram uma associação inversa e linear entre o consumo de produtos lácteos integrais e manteiga e a síndrome metabólica, e sugeriram que ácidos graxos encontrados nos laticínios poderiam ser responsáveis por esta associação 45 . No entanto, esses achados não têm sido confirmados em ensaios clínicos 46 . Devido à natureza transversal do presente estudo, não se pode descartar a possibilidade de 
causalidade reversa, uma vez que o consumo de manteiga, como fonte de gordura saturada, tem sido classicamente desencorajado para indivíduos com dislipidemias.

Em relação à inflamação, não foram encontradas associações entre o consumo de lácteos totais, fermentados, integrais e com reduzido teor de gordura e os valores de PCR. Os resultados de estudos disponíveis na literatura que investigaram o impacto do consumo de produtos lácteos nos biomarcadores inflamatórios são inconsistentes. Numa revisão sistemática mais recente com 52 estudos clínicos que investigaram a relação dos lácteos com inflamação, 32 apresentaram efeito anti-inflamatório, 19 efeito pró-inflamatório e 27 trabalhos sem evidência de efeito. Estratificando os resultados de acordo com o status de saúde dos indivíduos, demonstrou-se uma evidência mais forte de efeito anti-inflamatório naqueles com desordens metabólicas do que em indivíduos saudáveis ${ }^{18}$. Considerando que no presente trabalho foram excluídos os indivíduos diabéticos, e que a resistência à insulina tem um papel central na fisiopatologia da síndrome metabólica, o perfil da população do estudo pode ter influenciado o resultado.

Este estudo tem como ponto forte a análise da associação entre lácteos e distintos preditores de risco cardiovascular (perfil lipídico e marcador inflamatório), considerando diferentes subgrupos de lácteos (integrais, reduzido teor de gordura e fermentados), além dos diferentes alimentos lácteos separadamente (leite, iogurte, queijo e manteiga). Além disso, a análise foi realizada em uma grande amostra (4.031 homens e 5.341 mulheres), com ampla faixa etária e controlada por variáveis demográficas, de saúde e dietéticas relevantes. No entanto, a natureza observacional do estudo tem limitações a serem consideradas, uma vez que os achados podem resultar de confusão residual, apesar do ajuste extensivo para outros potenciais fatores de confusão. Além disso, o desenho transversal, com uma única medida do perfil lipídico e da PCR, não permite conclusões sobre a causalidade. Outra limitação diz respeito à ausência de biomarcadores objetivos da ingestão dos produtos lácteos. O QFA, método utilizado para a estimativa do consumo de lácteos, por ser uma avaliação retrospectiva e subjetiva da dieta, pode levar a uma super ou subnotificação da ingestão alimentar. Apesar das limitações do QFA, foram desenvolvidas estratégias de garantia e controle de qualidade dos dados de forma a minimizar o erro, como pré-teste e validação do instrumento, treinamento e certificação da equipe para a padronização da técnica de coleta 47 .

\section{Conclusão}

Em ambos os sexos, o maior consumo de lácteos foi associado a uma menor chance de apresentar valores aumentados de TG/HDL-C, um importante preditor de risco para DCV; no entanto, não foi observada associação dos lácteos com inflamação subclínica, medida pela PCR, e nem com os valores de LDL-C, considerado fator de risco para aterosclerose. Ainda, a associação entre lácteos e os valores de TG e HDL-C apresentou especificidades em relação ao sexo e ao conteúdo de gordura e grau de fermentação dos lácteos, sendo necessárias evidências de estudos longitudinais e de intervenção que elucidem as relações de causa-efeito dos diferentes tipos de lácteos no perfil lipídico e sua relação com eventos cardiovasculares. 


\section{Colaboradores}

A. G. Ribeiro contribuiu com a concepção e desenho do estudo, análise estatística, interpretação dos dados, redação do manuscrito e aprovação da versão final. J. G. Mill contribuiu com a revisão da qualidade dos dados, interpretação dos dados, redação do manuscrito e aprovação da versão final. S. M. A. Matos contribuiu com a revisão da qualidade dos dados, redação do manuscrito e aprovação da versão final. G. Velasquez-Melendez contribuiu com a interpretação dos dados, redação do manuscrito e aprovação da versão final. N. V. Cade contribuiu com a revisão crítica do conteúdo intelectual e aprovação da versão final. M. C. B. Molina contribuiu com a concepção e desenho do estudo, interpretação dos dados, redação do manuscrito e aprovação da versão final.

\section{Informações adicionais}

ORCID: Amanda Gomes Ribeiro (0000-0003-28622111); José Geraldo Mill (0000-0002-0987-368X); Sheila Maria Alvim Matos (0000-0003-2080-9213); Gustavo Velasquez-Melendez (0000-0001-83495042); Nágela Valadão Cade (0000-0001-6073504X); Maria del Carmen Bisi Molina (0000-00028614-988X).

\section{Agradecimentos}

O ELSA-Brasil foi apoiado pelo Ministério da Saúde (Departamento de Ciência e Tecnologia) e pelo Ministério da Ciência, Tecnologia e Inovação (Financiadora de Estudos e Projetos - FINEP e Conselho Nacional de Desenvolvimento Científico e Tecnológico - CNPq). Os autores agradecem à equipe e aos participantes do ELSA-Brasil por suas importantes contribuições e à Fundação de Amparo à Pesquisa do Espírito Santo (FAPES).

\section{Referências}

1. Pan American Health Organization. Deaths due to noncommunicable diseases in countries of the Americas. 2016. http://www.paho.org/ hq/index.php?option $=$ com_content\&view $=$ article \&id $=10169 \&$ Itemid $=41167 \&$ lang $=$ en (acessado em 09/Ago/2018).

2. Mendis S, Puska P, Norrving B, editors. Global atlas on cardiovascular disease prevention and control. Geneva: World Health Organization/ World Heart Federation/World Stroke Organization; 2011.

3. Ference BA, Ginsberg HN, Graham I, Ray KK, Packard CJ, Bruckert E, et al. Low-density lipoproteins cause atherosclerotic cardiovascular disease. 1. Evidence from genetic, epidemiologic, and clinical studies. A consensus statement from the European Atherosclerosis Society Consensus Panel. Eur Heart J 2017; 38:2459-72.

4. Faludi A, Izar M, Saraiva J, Chacra A, Bianco $\mathrm{H}$, Afiune Neto A, et al. Atualização da Diretriz Brasileira de Dislipidemias e Prevenção da Aterosclerose - 2017. Arq Bras Cardiol 2017; 109 (2 Suppl 1):1-76.

5. Austin MA, King MC, Vranizan KM, Krauss RM. Atherogenic lipoprotein phenotype. A proposed genetic marker for coronary heart disease risk. Circulation 1990; 82:495-506.

6. Yokoyama K, Tani S, Matsuo R, Matsumoto $\mathrm{N}$. Increased triglyceride/high-density lipoprotein cholesterol ratio may be associated with reduction in the low-density lipoprotein particle size: assessment of atherosclerotic cardiovascular disease risk. Heart Vessels 2019; 34:227-36.

7. Quispe R, Manalac RJ, Faridi KF, Blaha MJ, Toth PP, Kulkarni KR, et al. Relationship of the triglyceride to high-density lipoprotein cholesterol (TG/HDL-C) ratio to the remainder of the lipid profile: the Very Large Database of Lipids-4 (VLDL-4) study. Atherosclerosis 2015; 242:243-50.

8. Salazar MR, Carbajal HA, Espeche WG, Leiva Sisnieguez CE, Balbín E, Dulbecco CA, et al. Relation among the plasma triglyceride/highdensity lipoprotein cholesterol concentration ratio, insulin resistance, and associated cardiometabolic risk factors in men and women. Am J Cardiol 2012; 109:1749-53.

9. Kannel WB, Vasan RS, Keyes MJ, Sullivan LM, Robins SJ. Usefulness of the triglyceride-highdensity lipoprotein versus the cholesterolhigh-density lipoprotein ratio for predicting insulin resistance and cardiometabolic risk (from the Framingham Offspring Cohort). Am J Cardiol 2008; 101:497-501.

10. Da Silva MS, Rudkowska I. Dairy products on metabolic health: current research and clinical implications. Maturitas 2014; 77:221-8.

11. Shin S, Lee H-W, Kim CE, Lim J, Lee J, Kang D. Association between milk consumption and metabolic syndrome among Korean adults: results from the Health Examinees Study. Nutrients 2017; 9:e1102. 
12. Huo Yung Kai S, Bongard V, Simon C, Ruidavets J-B, Arveiler D, Dallongeville J, et al. Lowfat and high-fat dairy products are differently related to blood lipids and cardiovascular risk score. Eur J Prev Cardiol 2014; 21:1557-67.

13. Nilsen R, Høstmark AT, Haug A, Skeie S. Effect of a high intake of cheese on cholesterol and metabolic syndrome: results of a randomized trial. Food Nutr Res 2015; 59:27651.

14. The Emerging Risk Factors Collaboration. C-reactive protein, fibrinogen, and cardiovascular disease prediction. N Engl J Med 2012; 367:1310-20.

15. Syauqy A, Hsu C-Y, Rau H-H, Chao JC-J. Association of dietary patterns with components of metabolic syndrome and inflammation among middle-aged and older adults with metabolic syndrome in Taiwan. Nutrients 2018; 10:e143.

16. Panagiotakos DB, Pitsavos CH, Zampelas AD, Chrysohoou CA, Stefanadis CI. Dairy products consumption is associated with decreased levels of inflammatory markers related to cardiovascular disease in apparently healthy adults: the ATTICA study. J Am Coll Nutr 2010; 29:357-64.

17. Dugan CE, Aguilar D, Park Y-K, Lee J-Y, Fernandez ML. Dairy consumption lowers systemic inflammation and liver enzymes in typically low-dairy consumers with clinical characteristics of metabolic syndrome. J Am Coll Nutr 2016; 35:255-61.

18. Bordoni A, Danesi F, Dardevet D, Dupont D, Fernandez AS, Gille D, et al. Dairy products and inflammation: a review of the clinical evidence. Crit Rev Food Sci Nutr 2017; 57:2497525 .

19. Labonté M-È, Couture P, Richard C, Desroches S, Lamarche B. Impact of dairy products on biomarkers of inflammation: a systematic review of randomized controlled nutritional intervention studies in overweight and obese adults. Am J Clin Nutr 2013; 97:706-17.

20. Aquino EML, Barreto SM, Bensenor IM, Carvalho MS, Chor D, Duncan BB, et al. Brazilian Longitudinal Study of Adult Health (ELSABrasil): objectives and design. Am J Epidemiol 2012; 175:315-24.

21. Schmidt MI, Duncan BB, Mill JG, Lotufo PA, Chor D, Barreto SM, et al. Cohort profile: Longitudinal Study of Adult Health (ELSABrasil). Int J Epidemiol 2015; 44:68-75.

22. Molina MCB, Benseñor IM, Cardoso LO, Velasquez-Melendez G, Drehmer M, Pereira TSS, et al. Reprodutibilidade e validade relativa do Questionário de Frequência Alimentar do ELSA -Brasil. Cad Saúde Pública 2013; 29:379-89.

23. Sociedade Brasileira de Patologia Clínica/Medicina Laboratorial. Recomendações da Sociedade Brasileira de Patologia Clínica/Medicina Laboratorial para coleta de sangue venoso. 2a Ed. Barueri: Minha Editora; 2010.

24. Fedeli LG, Vidigal PG, Leite CM, Castilhos CD, Pimentel RA, Maniero VC, et al. Logística de coleta e transporte de material biológico e organização do laboratório central no ELSA -Brasil. Rev Saúde Pública 2013; 47:63-71.
25. Salazar MR, Carbajal HA, Espeche WG, Leiva Sisnieguez CE, March CE, Balbín E, et al. Comparison of the abilities of the plasma triglyceride/high-density lipoprotein cholesterol ratio and the metabolic syndrome to identify insulin resistance. Diab Vasc Dis Res 2013; 10:346-52.

26. Pearson TA, Mensah GA, Alexander RW, Anderson JL, Cannon RO, Criqui M, et al. Markers of inflammation and cardiovascular disease: application to clinical and public health practice: a statement for healthcare professionals from the Centers for Disease Control and Prevention and the American Heart Association. Circulation 2003; 107:499-511.

27. The IPAQ Group. Guidelines for data processing and analysis of the International Physical Activity Questionnaire (IPAQ): short and long forms. 2005. http://www.ipaq.ki.se (acessado em 09/Ago/2018).

28. Qin L-Q, Xu J-Y, Han S-F, Zhang Z-L, Zhao Y-Y, Szeto IM. Dairy consumption and risk of cardiovascular disease: an updated meta-analysis of prospective cohort studies. Asia Pac J Clin Nutr 2015; 24:90-100.

29. Guo J, Astrup A, Lovegrove JA, Gijsbers L, Givens DI, Soedamah-Muthu SS. Milk and dairy consumption and risk of cardiovascular diseases and all-cause mortality: dose-response meta-analysis of prospective cohort studies. Eur J Epidemiol 2017; 32:269-87.

30. Mena-Sánchez G, Babio N, MartínezGonzález MÁ, Corella D, Schröder H, Vioque J, et al. Fermented dairy products, diet quality, and cardio-metabolic profile of a Mediterranean cohort at high cardiovascular risk. Nutr Metab Cardiovasc Dis 2018; 28:1002-11.

31. Babio N, Becerra-Tomás N, MartínezGonzález MÁ, Corella D, Estruch R, Ros E, et al. Consumption of yogurt, low-fat milk, and other low-fat dairy products is associated with lower risk of metabolic syndrome incidence in an elderly Mediterranean population. J Nutr 2015; 145:2308-16.

32. Høstmark AT, Tomten SE. The Oslo Health Study: cheese intake was negatively associated with the metabolic syndrome. J Am Coll Nutr 2011; 30:182-90.

33. Fumeron F, Lamri A, Emery N, Bellili N, Jaziri R, Porchay-Baldérelli I, et al. Dairy products and the metabolic syndrome in a prospective study, DESIR. J Am Coll Nutr 2011; 30 (5 Suppl 1):454S-63.

34. Raziani F, Tholstrup T, Kristensen MD, Svanegaard ML, Ritz C, Astrup A, et al. High intake of regular-fat cheese compared with reduced-fat cheese does not affect LDL cholesterol or risk markers of the metabolic syndrome: a randomized controlled trial. Am J Clin Nutr 2016; 104:973-81.

35. Thorning TK, Raben A, Bendsen NT, Jørgensen $\mathrm{HH}$, Kiilerich P, Ardö Y, et al. Importance of the fat content within the cheese-matrix for blood lipid profile, faecal fat excretion, and gut microbiome in growing pigs. Int Dairy J 2016; 61:67-75. 
36. Kjølbæk L, Lorenzen JK, Larsen LH, Astrup A. Calcium intake and the associations with faecal fat and energy excretion, and lipid profile in a free-living population. J Nutr Sci 2017; 6:e50.

37. Zheng H, Yde CC, Clausen MR, Kristensen M, Lorenzen J, Astrup A, et al. Metabolomics investigation to shed light on cheese as a possible piece in the French paradox puzzle. J Agric Food Chem 2015; 63:2830-9.

38. Thorning TK, Bertram HC, Bonjour J-P, de Groot L, Dupont D, Feeney E, et al. Whole dairy matrix or single nutrients in assessment of health effects: current evidence and knowledge gaps. Am J Clin Nutr 2017; 105:1033-45.

39. Higurashi S, Ogawa A, Nara TY, Kato K, Kadooka Y. Cheese consumption prevents fat accumulation in the liver and improves serum lipid parameters in rats fed a high-fat diet. Dairy Sci Technol 2016; 96:539-49.

40. Brassard D, Tessier-Grenier M, Allaire J, Rajendiran E, She Y, Ramprasath V, et al. Comparison of the impact of SFAs from cheese and butter on cardiometabolic risk factors: a randomized controlled trial. Am J Clin Nutr 2017; 105:800-9.

41. Engel S, Tholstrup T. Butter increased total and LDL cholesterol compared with olive oil but resulted in higher HDL cholesterol compared with a habitual diet. Am J Clin Nutr 2015; 102:309-15.

42. Brassard D, Arsenault BJ, Boyer M, Bernic D, Tessier-Grenier M, Talbot D, et al. Saturated fats from butter but not from cheese increase HDL-mediated cholesterol efflux capacity from J774 macrophages in men and women with abdominal obesity. J Nutr 2018; 148:57380.
43. Pimpin L, Wu JHY, Haskelberg H, Del Gobbo L, Mozaffarian D. Is butter back? A systematic review and meta-analysis of butter consumption and risk of cardiovascular disease, diabetes, and total mortality. PLoS One 2016; 11:e0158118.

44. Mozaffarian D, Cao H, King IB, Lemaitre RN, Song X, Siscovick DS, et al. Trans-palmitoleic acid, metabolic risk factors, and new-onset diabetes in U.S. adults: a cohort study. Ann Intern Med 2010; 153:790-9.

45. Drehmer M, Pereira MA, Schmidt MI, Alvim S, Lotufo PA, Luft VC, et al. Total and full-fat, but not low-fat, dairy product intakes are inversely associated with metabolic syndrome in adults. J Nutr 2016; 146:81-9.

46. Drouin-Chartier J-P, Côté JA, Labonté M-È, Brassard D, Tessier-Grenier M, Desroches S, et al. Comprehensive review of the impact of dairy foods and dairy fat on cardiometabolic risk. Adv Nutr 2016; 7:1041-51.

47. Schmidt MI, Griep RH, Passos VM, Luft VC, Goulart AC, Menezes GMS, et al. Estratégias e desenvolvimento de garantia e controle de qualidade no ELSA-Brasil. Rev Saúde Pública 2013; 47:105-12. 
Abstract

Lipid abnormalities and subclinical systemic inflammation are associated with atherosclerosis and are used as markers of cardiovascular risk. Studies have suggested a possible beneficial effect of dairy products on cardiovascular health, but the results in lipid and inflammatory markers are still controversial. This study aimed to assess the association between consumption of dairy prod$u c t s$ and their different subgroups and C-reactive protein $(C R P), L D L$-cholesterol $(L D L-C)$, and triglyceride/HDL-cholesterol ratio (TG/HDL-C) in participants in the Longitudinal Study of Adult Health (ELSA-Brasil) $(n=9,372)$. Consumption of dairy products was assessed via a validated food frequency questionnaire and expressed as servings/day. Total consumption of dairy products was described in four categories ( $\leq 1$ serving/day to $>4$ servings/day). The associations were estimated via odds ratios $(\mathrm{OR})$, using the group with the lowest consumption ( $\leq 1$ serving/day) as the reference. The lowest ORs for TG/HDL-C in the multivariate model (0.70; 95\% CI: 0.55-0.90 in men; and 0.55; 95\%CI: 0.43-0.70 in women) were found in the group that consumed $>4$ servings day of dairy products. These results were supported by the inverse associations between different subgroups of dairy products and the TG/HDL-C ratio. No association was found between consumption of dairy products and their subgroups and $L D L-C$ and CRP. The results suggest a possible beneficial effect of dairy products on lipid profile, but longitudinal and intervention studies are needed to elucidate the effect mechanisms of different types of dairy products.

Dairy Products; Inflammation; Cardiovascular Diseases; Dyslipidemias

\section{Resumen}

Las anormalidades lipídicas e inflamación sistémica subclínica están asociadas con el proceso de arteriosclerosis, siendo utilizadas como marcadores de riesgo cardiovascular. Los estudios sugieren un posible efecto benéfico de los productos lácteos en la salud cardiovascular, pero los resultados en marcadores lipídicos e inflamatorios todavía son controvertidos. El objetivo de este estudio fue evaluar la asociación entre el consumo de productos lácteos y sus diferentes subgrupos y proteina $C$ reativa (PCR), $L D L$-colesterol $(L D L-C)$ y razón triglicéridos/HDL-colesterol (TG/HDL-C) en los participantes del Estudio Longitudinal de Salud del Adulto (ELSA-Brasil) $(n=9.372)$. El consumo de lácteos fue evaluado mediante un cuestionario de frecuencia alimentaria validado, y presentado en porciones/día. El consumo total de lácteos se describió en cuatro categorías ( $\leq 1$ porción/día $a>4$ porciones/día). Las asociaciones fueron estimadas mediante odds ratios (OR), utilizando el grupo de menor consumo ( $\leq 1$ porción/día) como referencia. Los menores valores de OR para TG/ HDL-C en el modelo multivariado (0,70; IC95\%: 0,55-0,90 en hombres; $y$ 0,55; IC95\%: 0,43-0,70 en mujeres) se encontraron en el grupo con consumo $>4$ porciones/día de lácteos totales. Estos resultados se apoyaron en las asociaciones inversas encontradas entre diferentes subgrupos de lácteos y la razón $T G / H D L-C$. No se encontró asociación entre consumo de productos lácteos y sus subgrupos y valores de $L D L-C y$ de PCR. Los resultados sugieren un posible efecto benéfico de los lácteos en el perfil lipídico, pese a que se necesitan evidencias de estudios longitudinales y de intervención que eluciden los mecanismos de efecto de los diferentes tipos de lácteos.

Productos Lácteos; Inflamación; Enfermedades Cardiovasculares; Dislipidemias
Recebido em 11/Fev/2019

Versão final reapresentada em 10/Jun/2019

Aprovado em 17/Jun/2019 